\title{
Genome Diagnostics: Novel Strategies for Measuring Value
}

\author{
Robin Z. Hayeems, PhD; Stephanie Luca, MA; Eleanor Pullenayegum, PhD; \\ M. Stephen Meyn, MD, PhD; and Wendy J. Ungar, PhD
}

\begin{abstract}
SUMMARY
Genetic testing technology is rapidly evolving with the growth of personalized medicine. While test evaluation typically relies on laboratory measures of performance, tests can be costly and analytically and ethically complex. A more fulsome consideration of value is warranted to inform adoption and appropriate use. Herein we describe a methodology for developing novel clinician- and patient-reported measures of clinical and personal utility, aiming to capture the informational value of genome diagnostic tests. Adhering to core measurement science principles and standards, our 4-step process includes (1) tool development through scoping reviews and stakeholder interviews and surveys; (2) tool validation through prospective cohort studies to establish construct validity, inter- and intra-rater reliability; (3) tool application using comparative effectiveness assessment to gauge the comparative value of different types of genetic tests; and (4) tool dissemination, leveraging existing partnerships with international stakeholders to spur additional validation studies, comparative effectiveness research, cost-effectiveness analysis, and evidence-informed policy.

A scoping review of the clinical utility literature informed the development of a preliminary 25-item index. Qualitative interviews with 35 clinicians further informed the definition of our utility construct, item content, and item importance. Stakeholder surveys with 113 clinicians enabled further feedback on item content, importance, sensibility, response, and scoring options. An 18-item tool, the "Clinician-reported Genetic testing Utility InDEx" (C-GUIDE), is now undergoing validation, while development work on the patient-reported measure of utility is underway.

A methodologically innovative approach to the development of stakeholderinformed and clinimetrically sound measures of value for personalized medicine tests will assist technology users and decision makers globally.
\end{abstract}

J Manag Care Spec Pharm. 2019;25(10):1096-1101

Copyright $\odot 2019$, Academy of Managed Care Pharmacy. All rights reserved.

$\mathrm{T}$ he potential for substantial medical and economic benefits of genome-wide sequencing (GWS) as a means to enhance personalized medicine across a broad range of therapeutic areas has generated much enthusiasm. Anticipated benefits include the replacement of multiple expensive genetic tests with a single low-cost genomic sequence, reduced morbidity in patients with high genetic risk through early detection and treatment, and selection of more effective medications., ${ }^{1,2}$ However, much remains to be learned about these promised outcomes. ${ }^{3,4}$ While a compelling evidence base demonstrates a superior diagnostic yield for GWS compared with conventional testing strategies, ${ }^{5,6}$ determining the full value of these technologies requires a set of metrics that extends beyond laboratorybased performance parameters. ${ }^{3,4}$ More specifically, there is little evidence that reflects on the clinical or personal value of GWS, that is, a direct link between test results and clinical decision making (i.e., "clinical utility") ${ }^{4}$ or between test results and the patient/family experience (i.e., "personal utility"). Increasingly, policymakers are seeking robust evidence that reflects on more comprehensive measures of value in order to make informed investment decisions that engage practitioners and patients. ${ }^{3,4,7}$

A methodology for comprehensively measuring the value of genome diagnostics is needed to (a) assess value from multiple perspectives using clinimetrically sound measurement tools; (b) enable comparative effectiveness research to determine the value of genome diagnostics in a wide range of health care settings and systems; and (c) track change in how value is defined and operationalized over time. Following is a summary of our progress towards addressing these needs (Figure 1).

\section{Methods}

\section{Tool Development}

The American College of Medical Genetics and Genomics (ACMG) characterizes the concept of clinical utility as a genetic test's "effect on diagnostic or therapeutic management, implications for prognosis, health and psychological benefits to patients and their relatives, and economic impact on healthcare systems. ${ }^{14}$ While this definition is debated, ${ }^{8}$ experts agree that clinical utility is a complex construct for which a standardized approach to measurement does not exist. While studies that reflect on specific aspects of clinical utility are abundant in the literature, ${ }^{9}$ they tend to be clinically siloed and use a wide range of outcome measures. This results in an evidence base that is difficult to synthesize and has limited generalizability, in turn creating barriers for decision makers tasked with developing evidence-informed policy.

In contrast, our methodology aims to generate standardized measures that reflect on the ACMG's notion of clinical utility from clinician and patient perspectives. ${ }^{4}$ Our emerging measures focus on the informational value generated by genome diagnostics. Unlike drugs and devices that may have a direct effect on health outcomes, diagnostics generate information and other benefits that can be processed or acted upon in ways that may affect health outcomes in the longer term, but the relationship between the test and the health outcome is indirect. ${ }^{10}$ As such, nonhealth-related benefits are essential to capture in the context of diagnostic genomics.

Adhering to core measurement science principles, ${ }^{11-13}$ we are in the process of validating the "Clinician-reported Genetic testing Utility InDEx" (C-GUIDE) and the "Patient-reported 


\section{FIGURE 1 Tool Development, Validation, Application, and Dissemination}

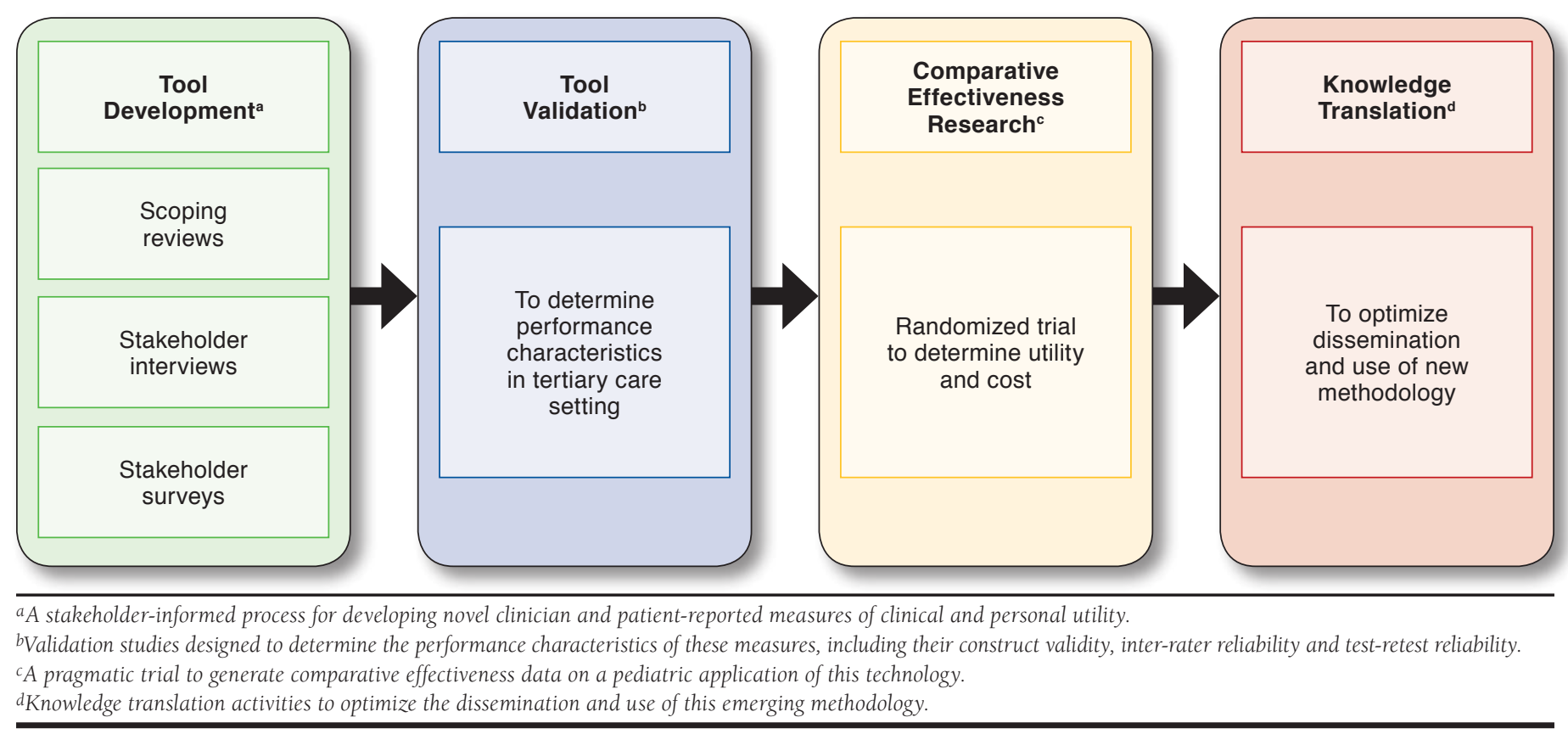

Genetic testing Utility inDEx" (P-GUIDE). ${ }^{14}$ These tools will enable clinicians and patients to assess the clinical and personal value of genetic testing, respectively. Designed with patient and clinician partners, our approach to measurement development includes the following 3 steps.

Step 1. Systematic Scoping Reviews. To optimize our selection of specific items for the C/P-GUIDE, systematic scoping reviews of the literature on the concepts of clinical/personal utility are required. Relevant studies were identified through citation databases (e.g., Ovid MEDLINE and Embase) using genetic testing and clinical/personal utility search terms. Drawing upon 149 studies that met inclusion criteria for clinical utility, ${ }^{9}$ we expanded the definition of this concept and identified 25 items that constituted the first draft of the C-GUIDE (Appendix, available in online article). ${ }^{9,14}$ A preliminary review of the personal utility literature suggests that relevant items include "helped me cope with my child's health risks" and "allowed me to locate social programs." A full scoping review of this concept, aligned with the previously mentioned methodology, ${ }^{15}$ is currently underway.

Step 2. Stakeholder Interviews. The preliminary version of the C-GUIDE was assessed by 35 clinician stakeholders who order genetic testing routinely. They were recruited as a convenience sample through local professional networks and snowball sampling. Feedback and an assessment of face validity was elicited using think-aloud methodology ${ }^{16}$ In a 45 -minute interview, participants were asked to define the concept of clinical utility, review the preliminary list of items, and provide feedback on/rate the importance of each item, using a 5-point scale. All interviews were transcribed verbatim. Item-specific feedback on content, wording, and domain assignment (e.g., diagnosis, medical management, and family implications) was coded using content analysis. Limitations of a convenience sample notwithstanding, the index was revised according to feedback and reduced to 20 items. ${ }^{14}$ Once we have established a literature-derived preliminary list of items for the P-GUIDE, we will invite parents/young adult patients involved in genetic testing within affiliated clinics to provide similar feedback.

Step 3. Stakeholder Surveys. To elicit further feedback on subsequent drafts of the C-GUIDE, 113 clinician stakeholders from Canada, the United States, and Australia completed an online survey. Respondents were asked to rank the importance of each index item and to rate the clinical sensibility of the tool overall. ${ }^{17}$ Where items ranked high and were qualitatively endorsed, they were retained in subsequent index drafts. An 11-member expert panel then provided additional quantitative and qualitative feedback on index items, domain assignment, item response options, and scoring strategies. A final 18-item version of the C-GUIDE is now undergoing validation..$^{14}$ Of note, for the purpose of validation, the C-GUIDE includes a "global item" of utility (i.e., "The genetic testing that my patient had prompted better care for my patient or his/her family overall"). 


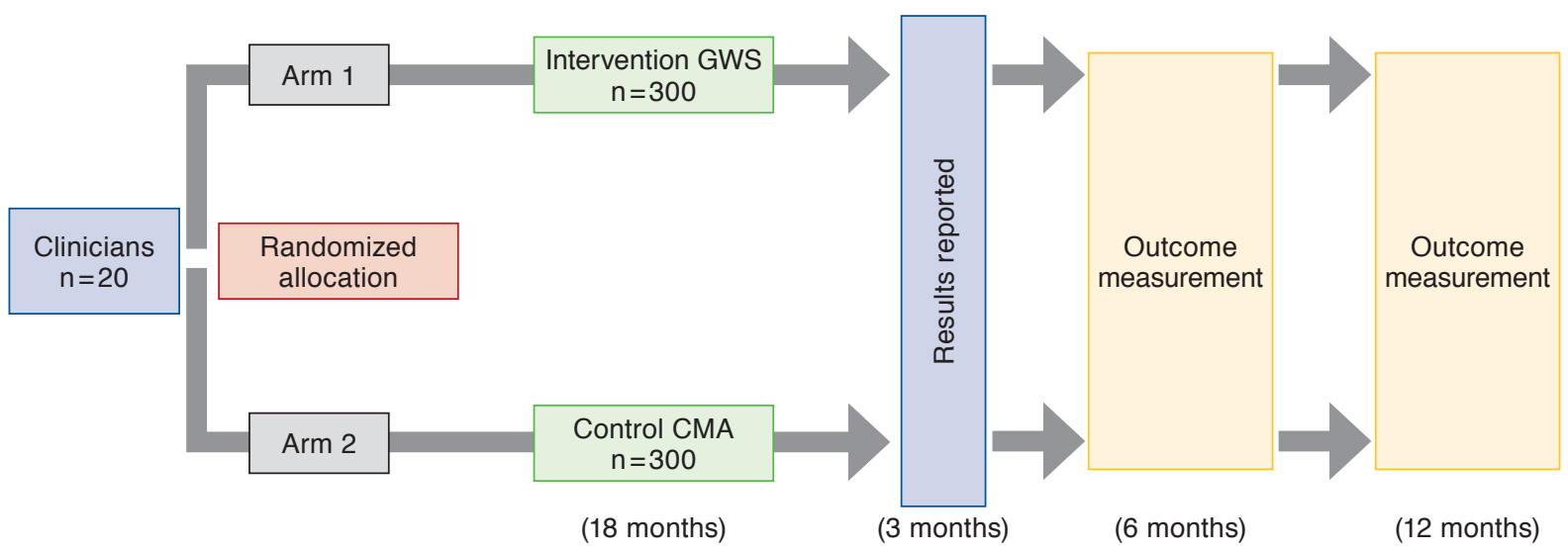

CMA = chromosome microarray; GWS = genome-wide sequencing.

For the P-GUIDE, we will leverage parents/young adult patients enrolled in Step 2 and expand our catchment to include additional clinical sites and family advisory networks affiliated with the Canadian Organization of Rare Disorders and the American-based National Organization for Rare Disorders. We aim to recruit approximately 200 parents/young adult patients into this development phase. Aligned with the approach taken for the C-GUIDE, we will conduct an online survey for parent/patient stakeholders and construct a panel of parent/patient experts to elicit feedback.

\section{Tool Validation}

Formal validation of the C-GUIDE, using accepted methods for assessing performance characteristics, including construct validity, test-retest reliability, and inter-rater reliability, is underway. ${ }^{11,18}$ The validation studies take place at The Hospital for Sick Children, the largest pediatric academic tertiary care center in Canada and a world leader in genomic medicine.

Sample and Recruitment. The C-GUIDE validation sample includes clinicians who routinely order GWS or conventional genetic tests (i.e., chromosome microarray [CMA] and gene panel tests) through the hospital's Genome Diagnostics Laboratory. Prospectively, clinicians will use the C-GUIDE to rate the utility of genetic testing for 200 children over an 18 -month period. Case selection will be stratified by test type, balanced between (a) GWS and (b) conventional genetic testing. Anticipating a conservative clinician response rate of $\sim 30 \%, 600$ ratings will be solicited to achieve the required sample $(n=200)$. The P-GUIDE validation sample will include parents of children/young adult patients who receive GWS or conventional test results as part of their clinical care (stratified as above). We will prospectively recruit 200 patient/parent participants over an 18-month period, inviting potential participants when they receive their or their child's test results.

Data Collection. To complete the C-GUIDE, clinicians will be asked to rely on their knowledge of the index case to assess the utility of the genetic test result received. Clinician respondents will complete the C-GUIDE as an online survey tool that requires them to select 1 of up to 3 categorical response options for each of 18 utility items presented on the index.

For the P-GUIDE, parents will be asked to reflect on their personal experience with genetic testing and, similarly using an online survey tool, select 1 response option for each of the set of P-GUIDE items that is developed. Participants will be asked to complete their respective scales within 1 week of reporting/receiving the genetic test result (Time 1). Since gold standard measures of clinical and personal utility for diagnostic tests do not exist, criterion validity cannot be assessed, but construct validity can be measured by examining the agreement between the C/P-GUIDE scores and the clinical/personal utility global scores that are embedded in the indices.

To determine intra-rater test-retest reliability, participants will complete the index 1 week following its initial completion (Time 2); agreement between scores at Time 1 and Time 2 will be determined for all cases. Inter-rater reliability will be assessed for the C-GUIDE using 18 hypothetical clinical vignettes and 11 members of our expert clinician panel. An important objective will be to determine the minimum clinically important difference for the novel measures; we will use the global indicator of change (GIC) anchor-based approach to achieve this objective. ${ }^{19}$ These data will be used to inform our parameters of utility for use in the following Tool Application section.

Analysis. The construct validity of the C-GUIDE (i.e., extent to which total item score agrees with a global score of utility) 
will be assessed by calculating the Pearson correlation between total C-GUIDE scores and the global item scores. Test-retest and inter-rater reliability will be measured using analysis of variance to generate intraclass correlation coefficients (ICC) comparing Time 1 and Time 2 scores, and between raters, respectively. To assess validity, it is desirable to have sufficient power to detect the presence of moderate to high levels of correlation between the C-GUIDE/P-GUIDE and the clinical/ personal global scores. Assuming there is a moderate correlation (>0.5), 200 participants enable $96 \%$ power to detect that this correlation is significantly different from 0.4. To assess inter-rater reliability, it is desirable to have sufficient power to detect the presence of moderate to high levels of agreement between the raters or time periods. A study that is designed to test the null hypothesis that the ICC is $<0.4$, assuming that the true ICC is 0.75 , requires a sample size of 35 to achieve $80 \%$ power at a one-sided significance level of 0.05 . We will be well powered to assess reliability and validity.

\section{Tool Application}

Following validation, we will apply these measures to real-time clinical environments to gauge the comparative value of different types of genome diagnostics. Having validated the C/PGUIDE in a pediatric tertiary care center, we will shift to measuring the utility of GWS compared with conventional genetic tests (i.e., CMA) in nontertiary pediatric care settings (i.e., community hospitals) where the uptake and perceived utility of new diagnostic tests may differ. In a cluster-randomized trial, we will directly compare the utility of GWS with CMA using the C/P-GUIDE and leverage the structure of the trial to capture health system impact and cost-effectiveness, enhancing our assessment of value with well-established methods. This will be the first comparative effectiveness study of pediatric GWS and will lay the methodological groundwork for an ongoing program of work that assesses the personal, clinical, and system value of genome diagnostics in a range of clinical settings and populations across global regions.

Sample and Recruitment. Over a period of $\sim 18$ months, we will recruit 600 children with developmental delay/intellectual disability (DD/ID) for whom genetic testing is indicated as part of a routine diagnostic work-up. Eight clinical sites in Ontario have agreed to participate in this trial; 4 sites will represent academic tertiary care centers ( $\mathrm{n}=10$ clinicians), and 4 sites will represent nonacademic community hospitals ( $\mathrm{n}=10$ clinicians). The clinical sites are similar in case mix, and all clinicians routinely order CMAs as a diagnostic test for children with DD/ID. Randomization will occur at the level of the clinician; each clinician will represent a cluster.

Data Collection. Participants from each site will follow a consistent workflow: baseline data collection, pretest counseling, clinical consultation, genomic analyses, disclosure of results, and outcome data collection. Genomic analyses and reporting procedures through the ordering clinician will follow established protocols. ${ }^{20,21}$ Genetic test results will be reported within 3 months of enrollment. Outcome data will be collected from the parents/young adult patients and clinicians within 1 month of disclosing CMA and GWS results, as well as 6 and 12 months later (Figure 2). Outcomes include C-GUIDE, P-GUIDE, and existing validated measures of health service utilization and cost effectiveness. ${ }^{22}$

Hypotheses include the following: (a) diagnostic GWS results will be associated with greater personal and clinical utility than CMA results; (b) diagnostic variants will be associated with greater utility among tertiary care clinicians compared with nontertiary care clinicians; and (c) GWS will be associated with a reduced rate (and cost) of posttest laboratory investigations and an increased rate (and cost) of posttest specialist consults, compared with CMA.

Analysis. To test these hypotheses, linear mixed effects models will be used to regress each outcome (e.g., C/P-GUIDE scores) onto the test received, with a random effect for the clinician. The coefficient of the variable for test type will indicate the difference in utility between GWS and CMA. A continuous variable representing health care utilization will be analyzed as a generalized linear mixed model with a Poisson link, as a function of test type and a random effect for clinician. We will also determine per patient costs associated with services used by multiplying the volume of resource use for each item by a corresponding current unit price in Canadian and U.S. dollars. Incremental cost of GWS per unit increase in diagnostic yield and per unit increase in clinical/personal utility compared with standard care will be determined.

Having determined the minimum clinically important difference for the novel measures during the validation studies, we will ensure we have adequate power to detect meaningful differences in this study. Using a validated patient-reported outcome measure that has been used in this population (i.e., state trait anxiety inventory) as a proxy for patient experience, our sample size is sufficient to achieve power $>0.8 .^{23}$

\section{Tool Dissemination}

While developing this methodology, we have generated awareness and interest in comprehensive outcomes-oriented research among stakeholders involved in genomic medicine in Canada, the United States, and internationally. Following tool validation, we will leverage existing partnerships with national and international stakeholder groups to disseminate our tools to patient communities, academic researchers, professional societies, and decision makers to spur additional validation studies, comparative effectiveness research, and evidence-informed policy.

To gain further traction for our methodology in the United States, we are in the early stages of pursuing endorsement through the National Quality Forum (NQF; http://www.qualityforum.org/Home.aspx). Mandated to achieve health care that 
is safe, effective, patient-centered, timely, efficient, and equitable, NQF has developed a portfolio of endorsed performance measures that can be used to quantify health care processes, outcomes, patient perceptions, and systems of high-quality care. Having followed NQF-endorsed best practices for measurement development, ${ }^{11}$ we will seek NQF endorsement for our novel measures, enabling their use by hospitals, health care systems, and government agencies for public reporting and quality improvement.

\section{Conclusions}

The proposed work described herein will be precedent setting in the field of personalized medicine. It will be methodologically innovative and rigorous in its development of stakeholderinformed and clinimetrically sound measurement tools for the field-tools that will be applicable to a wide range of health care settings and generalizable to health care systems globally.

\section{Authors}

ROBIN Z. HAYEEMS, PhD; ELEANOR PULLENAYEGUM, PhD; and WENDY J. UNGAR, PhD, The Hospital for Sick Children Research Institute and The University of Toronto, Toronto, Ontario, Canada. STEPHANIE LUCA, MA, The Hospital for Sick Children, Toronto, Ontario, Canada, and M. STEPHEN MEYN, MD, PhD, University of Wisconsin, Madison.

AUTHOR CORRESPONDENCE: Robin Z. Hayeems, PhD, The Hospital for Sick Children Research Institute and The University of Toronto, 686 Bay St., 11.9710, Toronto, Ontario M5G 0A4. Tel.: 416.813.7654, ext. 309259; E-mail: robin.hayeems@sickkids.ca.

\section{DISCLOSURES}

This work was supported by the Canadian Institutes of Health Research Operating Grant (\#PJT-152880) and the PhRMA Foundation Challenge Award. Publication of the study methodology or findings generated therein was not contingent on the sponsor's approval or censorship of the manuscript. The authors have nothing to disclose.

Results from this study were presented as a poster at the 40th Annual North American Meeting of the Society for Medical Decision Making; October 14, 2018; Montreal, QC; the Annual Meeting of the American Society of Human Genetics; October 18, 2018; San Diego, CA; and as an oral presentation at the Annual Meeting of the Canadian Association for Health Services and Policy Research; May 31, 2018; Montreal, QC.

\section{REFERENCES}

1. Manolio TA, Chisholm RL, Ozenberger B, et al. Implementing genomic medicine in the clinic: the future is here. Genet Med. 2013;15(4):258-67.

2. Wade C, Tarini BA, Wilfond BS. Growing up in the genomic era: implications of whole-genome sequencing for children, families, and pediatric practice. Annu Rev Genomics Hum Genet. 2013;14:535-55.

3. Caulfield T, Evans J, McGuire A, et al. Reflections on the cost of "lowcost" whole genome sequencing: framing the health policy debate. PLoS Biol. 2013;11(11):7-12
4. ACMG Board of Directors. Clinical utility of genetic and genomic services: a position statement of the ACMG. Genet Med. 2015;17(6):505-07.

5. Stavropoulos DJ, Merico D, Jobling R, et al. Whole-genome sequencing expands diagnostic utility and improves clinical management in paediatric medicine. NPJ Geno Med. January 13, 2016 [Epub]. Available at: https://www ncbi.nlm.nih.gov/pmc/articles/PMC5447450/. Accessed September 2, 2019.

6. Lionel AC, Costain G, Monfared N, et al. Improved diagnostic yield and comparable coverage to targeted gene sequencing panels suggests a role for whole-genome sequencing as a first-tier genetic test. Genet Med. 2018;20(4):435-43.

7. Kohler JN, Turbitt E, Lewis KL, et al. Defining personal utility in genomics: a Delphi study. Clin Genet. 2017;92(3):290-97.

8. Burke W1, Laberge AM, Press N. Debating clinical utility. Public Health Genomics. 2010;13(4):215-23.

9. Walcott S. Measuring the construct of clinical utility in the context of genetic testing: A scoping review [thesis]. Toronto, Ontario, Canada: University of Toronto; 2019. Available at: https://search.proquest.com/openv iew/6f6891752bdc62f758442f4659af9cfb/l?pq-origsite=gscholar\&cbl=18750 \&diss=y. Accessed September 2, 2019.

10. Bossuyt PMM, Reitsma JB, Linnet K, Moons KGM. Beyond diagnostic accuracy: the clinical utility of diagnostic tests. Clin Chem. 2012;58(12): 1636-43. Available at: http://clinchem.aaccjnls.org/content/58/12/1636.long. Accessed September 2, 2019

11. de Vet HCW, Terwee CB, Mokkink LB, Knol DL. Measurement in Medicine: A Practical Guide. Cambridge, UK: Cambridge University Press; 2011.

12. Centers for Medicare \& Medicaid Services. Blueprint for the CMS Measures Management System. Version 13.0. May 2017. Available at: https:// www.cms.gov/Medicare/Quality-Initiatives-Patient-Assessment-Instruments/ MMS/Downloads/Blueprint-130.pdf. Accessed September 2, 2019

13. Powers JH 3rd, Patrick DL, Walton MK, et al. Clinician-reported outcome assessments of treatment benefit: report of the ISPOR Clinical Outcome Assessment Emerging Good Practices Task Force. Value Health. 2017;20(1):2-14

14. Hayeems RZ, Luca S, Ungar WJ, et al. The development of the Clinicianreported Genetic testing Utility Index (C-GUIDE): a novel strategy for measuring the clinical utility of genetic testing. Genet Med. July 31, 2019 [Epub ahead of print]. Available at: https://www.nature.com/articles/s41436-0190620-0. Accessed September 2, 2019.

15. Arksey H, O'Malley L. Scoping studies: towards a methodological framework. Int J Soc Res Methodol. 2005;8(1):19-32. Available at: https://www. semanticscholar.org/paper/Scoping-studies\%3A-towards-a-methodologicalframework-Arksey-O\%27malley/f12499d98165f62f07f928f913bcl84albe6045c. Accessed September 2, 2019.

16. Van der Veer K, Hak T, Jansen H. The three-step test-interview (TSTI): an observation-based method for pretesting self-completion questionnaires. Surv Res Methods. 2008;2(3):143-50.

17. Rowe BH, Oxman AD. An assessment of the sensibility of a quality-oflife instrument. Am J Emerg Med. 1993;11(4):374-80.

18. McDowell I, Newell C. Measuring Health: A Guide to Rating Scales and Questionnaires. 2d ed. New York: Oxford University Press; 1996.

19. Engel L, Beaton DE, Touma Z. Minimal clinically important difference: a review of outcome measure score interpretation. Rheum Dis Clin North Am. 2018;44(2):177-88.

20. Bowdin S, Ray PN, Cohn RD, Meyn MS. The genome clinic: a multidisciplinary approach to assessing the opportunities and challenges of integrating genomic analysis into clinical care. Hum Mutat. 2014;35(5):513-19.

21. Bowdin SC, Hayeems RZ, Cohn RD, Meyn MS. The SickKids Genome Clinic: developing and evaluating a pediatric model for individualized genomic medicine. Clin Genet. 2016;89(1):10-19. 
22. Ungar WJ, Tsiplova K, Millar N, Smith IM. Development of the Resource Use Questionnaire (RUQ-P) for families with preschool children with neurodevelopmental disorders: validation in children with autism spectrum disorder. Clin Pract Pediatr Psychol. 2018;6(2):164-78.

23. Wakefield CE, Hanlon LV, Tucker KM, et al. The psycholgoical impact of genetic information on children: a systematic review. Genet Med. 2016;18(8):755-62. 


\section{APPENDIX First Draft of C-GUIDE}

"The genetic testing that my patient had..."

1. Confirms findings from other diagnostic tests

2. Establishes a diagnosis for my patient

3. Halts my patient's diagnostic odyssey

4. Helps to define my patient's prognosis

5. Alters my differential diagnosis

6. Rules out potential diagnoses that were in my differential

7. Identifies a new health risk for my patient that may manifest in the future

8. Indicates that further diagnostic investigations are required

9. Suggests that an invasive procedure can be avoided

10. Suggests that a specialist referral is indicated

11. Suggests that a specific surveillance protocol is indicated

12. Suggests that a referral to an allied health professional is indicated (e.g. PT, OT, speech)

13. Enables my patient to access health/social services more quickly

14. Reveals that a medication alteration is indicated (stoppage, altered dose, altered drug)

15. Identifies a medication-related risk

16. Identifies a medication-related protective effect

17. Reveals that a specific treatment is indicated

18. Enables me to provide anticipatory guidance for my patient related to a future health risk

19. Enables me to inform my patient about a specific preventive health measure or behavior

20. Assists with identifying a support group for my patient or his/her family

21. Identifies a health risk in my patient's family members

22. Identifies a reproductive risk in my patient's family members

23. Identifies a medication-related risk or protective effect in my patient's family members

24. Reveals that genetic testing for a family member is indicated

25. Imposes risk on the patient or family related to insurance or employment discrimination

C-Guide=Clinician-reported Genetic testing Utility InDEx; OT = occupational therapy; PT = physical therapy. 